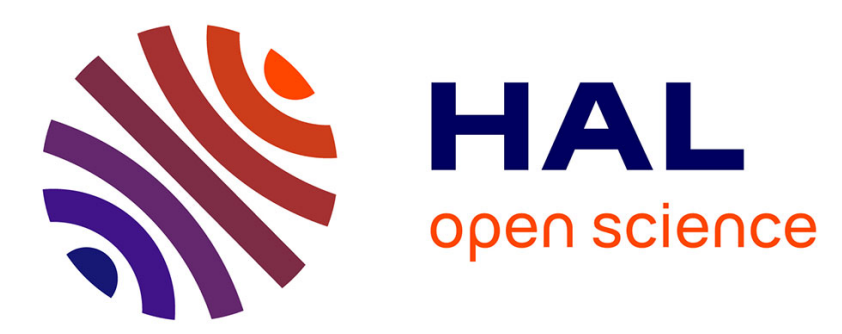

\title{
Study of self-avatar's influence on motor skills training in immersive virtual environments
}

\author{
Aylen Ricca, Amine Chellali, Samir Otmane
}

\section{To cite this version:}

Aylen Ricca, Amine Chellali, Samir Otmane. Study of self-avatar's influence on motor skills training in immersive virtual environments. 2018 Virtual Reality International Conference - Laval Virtual

(VRIC 2018), Apr 2018, Lavel, France. pp.1-4, 10.1145/3234253.3234304 . hal-01968252

\section{HAL Id: hal-01968252 \\ https://hal.science/hal-01968252}

Submitted on 8 Feb 2021

HAL is a multi-disciplinary open access archive for the deposit and dissemination of scientific research documents, whether they are published or not. The documents may come from teaching and research institutions in France or abroad, or from public or private research centers.
L'archive ouverte pluridisciplinaire HAL, est destinée au dépôt et à la diffusion de documents scientifiques de niveau recherche, publiés ou non, émanant des établissements d'enseignement et de recherche français ou étrangers, des laboratoires publics ou privés. 


\section{Study of self-avatar's influence on motor skills training in immersive virtual environments}

\author{
Aylen Ricca \\ IBISC, Univ Evry, Université \\ Paris-Saclay \\ Evry, France \\ aylen.ricca@ibisc.fr
}

\author{
Amine Chellali \\ IBISC, Univ Evry, Université \\ Paris-Saclay \\ Evry, France \\ amine.chellali@ibisc.fr
}

\author{
Samir Otmane \\ IBISC, Univ Evry, Université \\ Paris-Saclay \\ Evry, France \\ samir.otmane@ibisc.fr
}

\begin{abstract}
Immersive virtual reality simulators are increasingly being used in different domains. One open research problematic of such systems is the user's representation inside the environment, what is called, the self-avatar representation. Our research focus on using immersive virtual environments (IVEs) for training motor skills. In this case, the typical self-avatar representation is the user's hand. Researchers have focused on studying the influence of hand appearance and structure on the user's sense of presence and embodiment, but little is known about the role it plays in learning motor skills.

In this paper we present the design and implementation of a virtual reality (VR) training simulator for a basic motor task, as well as the design of an experiment to investigate this research problematic.
\end{abstract}

\section{CCS CONCEPTS}

- Human-centered computing $\rightarrow$ Human computer interaction (HCI); User studies; Virtual reality;

\section{KEYWORDS}

Learning curve study, motor skill training, self-avatar representation, virtual simulation.

\section{INTRODUCTION}

Immersive virtual reality technology allows to create realistic simulations as an alternative for real world situations which can be either dangerous, almost impossible to reproduce or expensive to carry out. For instance, in the aviation domain, pilots can be trained in virtual environments (VE), to perform complicate and risky maneuvers, and landings under different weather conditions prior to real flights [6]. Automation industry can use immersive virtual environments (IVEs) to real-size models and validate ergonomic, security and design choices without the need for building a physical prototype [8]. Another example of IVEs use is skills training in maintenance industry, such as parts assembly and machine repair [18]. Finally, IVEs are promising in medical education, where students and practitioners can benefit from patient-safety training, as well as from practicing complex surgical procedures or experiencing unexpected operating room complications [7].

IVEs that use head-mounted displays (HMD) allow users to explore the virtual scenario from a first person perspective, and be completely surrounded by it. In this context, one open research issue is self-avatar representation, and its influence on user's perception and interaction behavior within the virtual environment. The self-avatar is the visual representation of the user's body inside a 3D environment [15]. In motor skills training, such as assembling of mechanical parts, instrument handling, or tying knots in surgery, the avatar representation concerns primarily the user's hands.

Research has focused on studying the influence that hand appearance and structure may have on the user's sense of presence and embodiment inside the virtual environment.

Through this ongoing research, we want to move further by studying the implications that user's avatar may have on the learning process of a motor skill which requires tool handling, in an IVE. Our research objective is to determine whether the visual representation of the hands movements affects the learning curve of a motor skill training task, and whether the tool visualization is enough due to perceiving it as a continuity of the user's hands. Furthermore, we also want to explore whether seeing the hand movements impacts the transfer of the learned skill to the real-world setup. To address this problematic, we present in this paper the design and implementation of a VR training simulator for a basic motor task. We also present the design of an experiment being currently conducted in order to investigate our research questions.

\section{RELATED WORK}

The importance of body-based perception in immersive environments has been studied for some time [20] [3]. Usoh et al., during the study of alternate ways to control locomotion in a VE, concluded in 1999 that there is a high correlation between presence and the degree of association with the virtual body [21] (sense of embodiment). Kilteni et al. defines the sense of embodiment towards a body B as the sense that emerges when B's properties are processed as if they were properties of one's own biological body [14]. Embodiment can be decomposed into three dimensions, the sense of self-location, the sense of agency, and the sense of body ownership [13]. Self-location defines the space where user perceives he/she is located, providing a frame of reference for his/her body and determining the place where sensations are registered. The sense of agency exists when user perceives that he/she is in control of his/her own actions within the environment. Finally, ownership can be defined as the user's feeling that the artificial body is experienced as his/her body, and is the source of sensations [12]. Related to this subject, Argelaguet et al. recent results showed that the sense of agency relates to the virtual hand control, and ownership to the visual appearance of the virtual hand [1]. In their experiment, they compared three different visual representations of user's hand by keeping the same control mechanism.

Experiments based on the rubber hand illusion, initially developed in 1998 [4], show that if the user is presented with a conflict between visual position and proprioceptive position of his hand, 
he/she will strongly accept it as being where it is seen, so accepting the visual over the proprioceptive position [16]. González-Franco et al. investigated brain activity in response to pain observation when the user's virtual hand was attacked with a knife [11]. The results allowed to conclude that when a user is in an IVE, he/she has body ownership towards his/her body representation, up to the point of experiencing observed events to take place in reality. Hoyet et al. also performed a study on the hand representation, but focusing on how users perceive a six-digit realistic virtual hand. They concluded that users responses towards controlling a six-digit hand despite the structural difference was positive, being able to accept, up to an extent, this representation as their own.

Human motor skills can be influenced by visual feedback in general and particularly from the body of the user (user's avatar). For instance, Ossmy et al. studied how the size of the virtual hand affected short-term motor skill learning [17]. The task in their experiment consisted of learning a sequence of finger movements. The results showed that performance increased with a larger size of the virtual hand, and when the ratio of it and the real hand was close to 1 . Moreover, these results were observed only when the hand was controlled by the user, and not when the user was simply a spectator during training. This suggest an importance of the integration of visual and tactile human sensors. In this matter, Mohier et al. results show that performance on distance estimations were higher with the presence of an avatar when it was animated in correspondence with the user's real body movements [16].

On the other hand, Lok et al. studied the effects of interacting with real objects and the self-avatar fidelity on a block arrangement task [15]. They concluded that the kinematic fidelity of the avatar (movement) is significantly more important than the visual fidelity (appearance) for increasing the sense of presence within the virtual environment, and that avatar fidelity did not affect performance on this manual task. Finally, Van Nguyen et al. also explored the influence of a virtual hand in user's feeling of accuracy and sense of realism for a needle insertion trainer [22]. The results showed that the static virtual hand increased the feeling of accuracy, but not the overall realism of the environment, which can be possibly explained by the fact that the virtual hand was a non-animated model, hence, not reproducing user's real position and movements.

As we can see from recent work, self-avatar has been studied to explain its influence on presence and embodiment in general tasks. We want to grow the existence knowledge by focusing on the self-avatar impact on the learning curve of motor skills involving tool manipulation in IVEs. Therefore, we have designed and implemented a VR simulator and an experimental protocol which can be used to answer this research problematic.

\section{SIMULATOR DESIGN AND IMPLEMENTATION}

To investigate our research question, we have designed and developed a virtual reality simulator for training a basic motor skill task in minimally invasive surgery. In fact, virtual simulators could have an important impact on training in this application domain.

The basic motor task chosen for our simulator is a pick-andplace task, and is designed so as the user must use one tool in each hand to manipulate small objects from one position to another, by

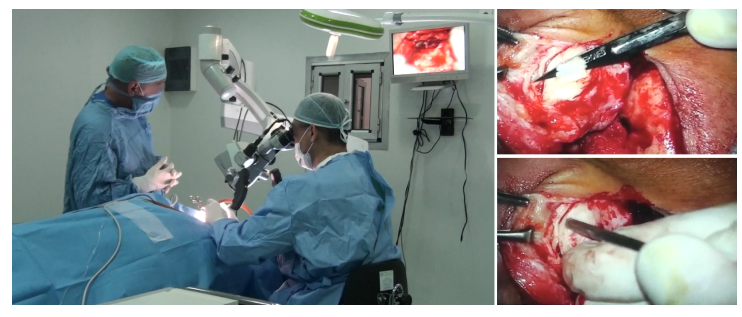

Figure 1: Ear surgery: Surgeon performing the surgery (left), images visualized through microscope (right).

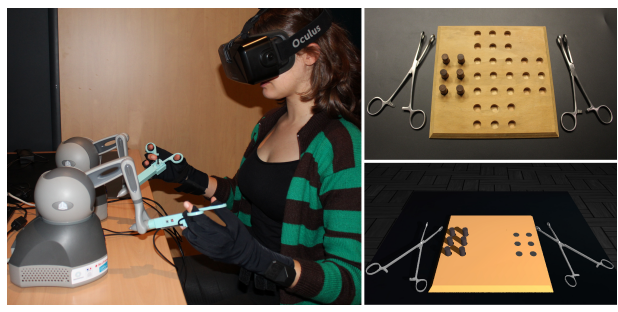

Figure 2: Simulator implementation

switching them from one tool to the other. It is inspired from the peg and transfer task used in VR simulators to train surgeons to master hand-eye coordination and bi-manual dexterity in laparoscopic surgery [9]. The difference between the chosen task and the original laparoscopic training task is that users see the objects in a $3 \mathrm{D}$ view and in the same direction where they manipulate them. This is similar to what can be experienced in ear surgery under a microscope, for instance (see Figure 1).

In addition, preview research has shown that haptic feedback is an important requirement for surgical VR trainers. Indeed, surgeons must rely on their haptic skills when interacting with patient's tissues and organs to avoid fatal mistakes [2] [23]. Taking this into account, the designed prototype includes haptic interfaces to render the necessary feedback.

Following our design choices, we developed a prototype of our simulator using $\mathrm{C}++$. It uses the Chai3d framework for haptic force feedback simulation and ODE physics engine for collisions detection. This simulator includes two haptic devices (Geomagic Touch desktop with 3DoF for force feedback -forces $<3 \mathrm{~N}-$ and $6 \mathrm{DoF}$ on position) with a handle of a forceps tool prototype attached (interface). Participants are immersed in the virtual environment through an Oculus Rift, which provides a first-person perspective, and though provoking a stronger sense of self-location [19]. In order to reproduce the user's real hands movement, a pair of 5DT data gloves are employed, which allow for fingers posture position while the user is holding the tool interface (see Figure 2). The virtual environment consists of small pickable objects in the form of cylinders, virtual graspers representing the tools, and the hand representation. In addition, a physical simulator is used for comparison with the virtual trainer. It consists of real objects and tools.

\section{EXPERIMENTAL DESIGN}

In this section, we present the design of our experimental protocol. The main goal is to gain insight in the importance of user's 3D 
avatar inside a VR trainer for learning a motor skill. Specifically, it aims to explore how the user's real hand movement representation can influence the learning curve of a motor task requiring the manipulation of a tool, and its transfer to the real world task. In addition, it allows validating the simulator as an effective trainer. More particularly, the research questions to explore are:

(1) does the representation of the users' hands movements influences the learning process of a motor skill?

(2) does the representation of the users' hands movements permits better transfer to the real world task?

(3) do users think they have a better control of the system and a higher sense of ownership when the real movements of their hands are reproduced?

To address these questions, the designed experiment consists of participants being asked to train themselves in a VR simulator and perform a final evaluation test in a physical environment. Our hypotheses are:

- Face validity:

- H1 Training in the virtual reality simulator improves user's task performance.

- Transfer to the real world:

- H2 Training in the virtual reality simulator impacts positively on the real-task users' performance.

- Influence of the hand representation:

- H3 Users' performance is higher when they have the real hand representation compared to the no hand representation condition.

- H4 Users in the real hand movement condition feel a higher sense of ownership than users in the no hand representation condition.

- H5 Users in the real hand movement condition feel a higher sense of agency than users in the no hand representation condition.

\subsection{Experimental design}

The experiment will follow a between subjects design with 1 factor (training group) with 3 levels: training with the presence of the user's virtual hand representation (group 1), training without the presence of the user's virtual hand representation (group 2), and a control group without training. The dependent variables are the user's performance, i.e. the task completion time, the virtual objects manipulation accuracy, and the number of errors.

\subsection{Procedure}

This study was reviewed and approved by an institutional ethical review committee. The experimental procedure is divided into five sessions (see Figure 3), which are described hereafter.

User's participation starts with a consent form signature and a subject information sheet used to collect demographic information.

4.2.1 Familiarization session: Participants are exposed to the experimental setup in order to gain familiarity with the environment, interfaces and interactions to use. This phase guarantees that all users had a first contact with the simulator and understand how to interact with it to perform the experimental task.
4.2.2 Pre-test session: All the participants are asked to perform the experimental task 3 times on the physical prototype and 6 times on the virtual one, 3 times for each training condition. They must also answer a comparative presence state questionnaire (based on literature embodiment questionnaires [1]) for both conditions.

This session records the initial performance level for each user. It is done on both, physical and virtual simulators, to compare performance with the post-test (face-validity and transfer validation).

4.2.3 Training sessions: Concern only groups 1 and 2, and starts after the pre-test. Participants are asked to perform the task in the virtual environment as many times as possible during 20 minutes. Session duration was defined by considering the fact that users wear a HMD during the task. In fact, it is necessary to find a balance between the practice time and the participants' fatigue. Training session is repeated 5 days each week during 2 weeks. The virtual environment in which to train depends on the training condition.

4.2.4 Post-test session: Is the last phase of a typical learning curve study and allows to study performance results after training. It is performed after the last training session (groups 1 and 2), or after 15 days (control group). It is important to note that this session should not be performed immediately after the last training session so as to avoid that participants feel tired and distort the results.

All the participants are asked to perform the experimental task 3 times on the physical prototype and 6 times on the virtual one, 3 times for each training condition.

4.2.5 Retention session: This session permits to study the retention of the learned skill sometime after the post-test took place. It could provide better conclusions on the effectiveness of the simulator and the knowledge transfer.

All participants are asked to perform the experimental task 3 times on the physical prototype and 6 times on the virtual one, 3 times for each training condition. At the end, they answer the system usability (SUS) questionnaire [5], used to gain insight in the design choices for following iterations.

\subsection{Measures}

As mentioned before, performance measures are the task completion time (ms), the accuracy of objects' placement (mm), and the number of errors (counted as objects dropped during trials). In addition to the quantitative measures, participants are asked to answer the System Usability Questionnaire (SUS) at the end of the experiment [5], and a comparative questionnaire for the sense of presence and embodiment for each condition in the virtual simulator (based on the one presented in [1]). Finally, user's hand trajectories are recorded in order to study the different behaviors of both training groups. In this matter, the strategy is to analyze the mean trajectory for each user and compare them in terms of minimal length.

\subsection{Participants}

A minimum of 18 participants older than 18 years old from both genders will be recruited for this experiment. The minimum number of participants to obtain a satisfactory statistical strength was calculated based on the method of Cohen (1988) [10]. Thus, the minimum number of subjects is estimated to 6 per condition. The recruitment targets people who may or not have prior experience 


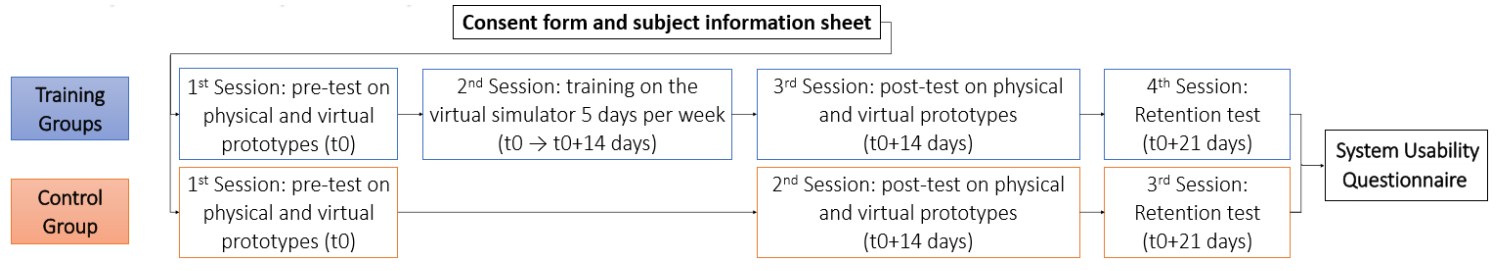

Figure 3: Experimental protocol summary.

with VR technologies or video games. Subjects who feel uncomfortable wearing HMD, or present motor problems on their upper extremities can not be included in this study.

\section{CONCLUSION AND FUTURE WORK}

In this paper we present the design of a VR simulator and an experimental protocol to study the influence of self-avatar representation on the learning curve of a motor skill training in IVEs.

The experimental protocol is exemplified with the learning of a pick and place motor task, but it could be applied to any task involving the training of a motor skill, in which users' hands wear tools. We focus on medical skills training, as being today one area of open research. Many are the simulators that exist in the market, but little is known of their effectiveness for training motor skills.

We plan to evaluate the developed VR simulator through the presented experimental protocol in order to gain insight on this research problematic. Firstly, the results will determine if the simulator serves it purpose as a trainer for the motor skill, and the learning curve analysis may thrown insights into its efficiency. Moreover, the difference between the training conditions performance, if it exists, will allow to justify or not the use of the hand representation in addition to the representation of the manipulation tool. This fact is important from an affordable point of view, because tracking systems to detect user's hands movements are expensive and add a complication factor to the development of VR simulators. All things considered, the evaluation results will allow to determine up to which point VR trainers should replicate reality to guarantee the learning of motor skills like the one presented in this research.

\section{ACKNOWLEDGEMENTS}

This research work was partially supported by the Paris Ile-deFrance Region.

\section{REFERENCES}

[1] Ferran Argelaguet, Ludovic Hoyet, Michaël Trico, and Anatole Lécuyer. 2016. The role of interaction in virtual embodiment: Effects of the virtual hand representation. In Virtual Reality (VR), 2016 IEEE. IEEE, 3-10.

[2] Cagatay Basdogan, Suvranu De, Jung Kim, Manivannan Muniyandi, Hyun Kim, and Mandayam A Srinivasan. 2004. Haptics in minimally invasive surgical simulation and training. IEEE computer graphics and applications 24, 2 (2004), $56-64$

[3] Frank Biocca. 1997. The cyborg's dilemma: Progressive embodiment in virtual environments. Fournal of Computer-Mediated Communication 3, 2 (1997), 0-0.

[4] Matthew Botvinick and Jonathan Cohen. 1998. Rubber hands 'feel' touch that eyes see. Nature 391, 6669 (1998), 756.

[5] John Brooke et al. 1996. SUS-A quick and dirty usability scale. Usability evaluation in industry 189, 194 (1996), 4-7.

[6] Frederick P Brooks. 1999. What's real about virtual reality? IEEE Computer graphics and applications 19, 6 (1999), 16-27.
[7] CE Buckley, E Nugent, D Ryan, and PC Neary. 2012. Virtual reality-A new era in surgical training. In Virtual Reality in Psychological, Medical and Pedagogical Applications. InTech.

[8] Amine Chellali, Frederic Jourdan, and Cédric Dumas. 2013. VR4D: An immersive and collaborative experience to improve the interior design process. In 5 th foint Virtual Reality Conference of EGVE and EuroVR, JVRC 2013. 61-65.

[9] Amine Chellali, Helena Mentis, Amie Miller, Woojin Ahn, Venkata S Arikatla, Ganesh Sankaranarayanan, Suvranu De, Steven D Schwaitzberg, and Caroline GL Cao. 2016. Achieving interface and environment fidelity in the Virtual Basic Laparoscopic Surgical Trainer. International Journal of Human-Computer Studies 96 (2016), 22-37.

[10] Jacob Cohen. 1988. Statistical power analysis for the behavioral sciences. Hilsdale. Nf: Lawrence Earlbaum Associates 2 (1988)

[11] Mar González-Franco, Tabitha C Peck, Antoni Rodríguez-Fornells, and Mel Slater. 2014. A threat to a virtual hand elicits motor cortex activation. Experimental brain research 232, 3 (2014), 875-887.

[12] Ludovic Hoyet, Ferran Argelaguet, Corentin Nicole, and Anatole Lécuyer. 2016. "Wow! I have six Fingers!": Would You accept structural changes of Your hand in Vr? Frontiers in Robotics and AI 3 (2016), 27.

[13] Konstantina Kilteni, Raphaela Groten, and Mel Slater. 2012. The sense of embodiment in virtual reality. Presence: Teleoperators and Virtual Environments 21, 4 (2012), 373-387.

[14] Konstantina Kilteni, Jean-Marie Normand, Maria V Sanchez-Vives, and Mel Slater. 2012. Extending body space in immersive virtual reality: a very long arm illusion. PloS one 7, 7 (2012).

[15] Benjamin Lok, Samir Naik, Mary Whitton, and Frederick P Brooks. 2003. Effects of handling real objects and self-avatar fidelity on cognitive task performance and sense of presence in virtual environments. Presence: Teleoperators and Virtual Environments 12, 6 (2003), 615-628.

[16] Betty J Mohler, Sarah H Creem-Regehr, William B Thompson, and Heinrich H Bülthoff. 2010. The effect of viewing a self-avatar on distance judgments in an HMD-based virtual environment. Presence: Teleoperators and Virtual Environments 19, 3 (2010), 230-242

[17] Ori Ossmy and Roy Mukamel. 2017. Short Term Motor-Skill Acquisition Improves with Size of Self-Controlled Virtual Hands. PloS one 12, 1 (2017).

[18] Matthieu Poyade. 2013. Motor skill training using virtual reality and haptic interaction -A case study in industrial maintenance. Ph.D. Dissertation. University of Malaga.

[19] Mel Slater, Bernhard Spanlang, Maria V Sanchez-Vives, and Olaf Blanke. 2010. First person experience of body transfer in virtual reality. PloS one 5, 5 (2010).

[20] Mel Slater and Martin Usoh. 1994. Body centred interaction in immersive virtua environments. Artificial life and virtual reality 1, 1994 (1994), 125-148.

[21] Martin Usoh, Kevin Arthur, Mary C Whitton, Rui Bastos, Anthony Steed, Mel Slater, and Frederick P Brooks Jr. 1999. Walking > walking-in-place> flying, in virtual environments. In Proceedings of the 26th annual conference on Computer graphics and interactive techniques. ACM Press/Addison-Wesley Publishing Co., 359-364.

[22] Duc Van Nguyen, Safa Ben Lakhal, and Amine Chellali. 2015. Preliminary evaluation of a virtual needle insertion training system. In Virtual Reality (VR), 2015 IEEE. IEEE, 247-248.

[23] M Zhou, S Tse, A Derevianko, DB Jones, SD Schwaitzberg, and CGL Cao. 2012. Effect of haptic feedback in laparoscopic surgery skill acquisition. Surgical endoscopy 26, 4 (2012), 1128-1134. 